\title{
Unresolved sources in the Galactic diffuse gamma-ray emission at TeV energies
}

\author{
Kathrin Egberts* \\ Institut für Physik und Astronomie, Universität Potsdam, Karl-Liebknecht-Strasse 24/25, D \\ 14476 Potsdam, Germany \\ E-mail: kathrin.egbertseuni-potsdam.de
}

Diffuse $\gamma$-ray emission is a viable tool to probe cosmic rays and their propagation in the Milky Way. In the $\mathrm{MeV}$ to $\mathrm{GeV}$ energy range the Galactic diffuse emission has been explored in great detail by Fermi-LAT. At very high energies (VHE), $\gamma$-ray sources dominate the TeV sky. Any VHE large-scale diffuse emission is thus likely to consist to a large fraction of $\gamma$-ray sources that are unresolved due to limited instrument sensitivity and the resulting inability to resolve the Milky Way to its full depth. The H.E.S.S. experiment has performed a first measurement of Galactic diffuse $\gamma$-ray emission at $\mathrm{TeV}$ energies outside of known $\gamma$-ray source regions. Interpretation of such a signal requires disentangling of the contributions of "truly diffuse" emission stemming from cosmic-ray propagation and the emission associated with unresolved sources. This can only be achieved by invoking model assumptions for both contributions. While sophisticated codes for cosmic-ray propagation already exist, unresolved-source emission has long been neglected due to its little impact at $\mathrm{MeV}$ to $\mathrm{GeV}$ energies. In this contribution first steps towards an estimation of the emission of unresolved $\gamma$-ray sources at $\mathrm{TeV}$ energies are presented. A VHE $\gamma$-ray source population is simulated, which follows the spiral structure of the Milky Way. The simulations are constrained by the sum of the detected Galactic VHE $\gamma$-ray sources in the range of completeness and the accumulated $\gamma$-ray emission of this population is compared to the measurement of diffuse $\mathrm{TeV}$ emission.

35th International Cosmic Ray Conference - ICRC2017

10-20 July, 2017

Bexco, Busan, Korea

*Speaker. 


\section{Introduction}

Cosmic rays permeate our Galaxy and while doing so they interact and produce diffuse $\gamma$-rays. The main interaction mechanisms resulting in these $\gamma$-rays are for the case of cosmic-ray electrons bremsstrahlung and inverse Compton scattering, for the case of hadronic cosmic rays the production and decay of neutral pions in the interaction with interstellar matter. The measurements of diffuse $\gamma$-rays can therefore be used to study the distribution and along with that the propagation of cosmic rays in the Milky Way. Such measurements have been performed at $\mathrm{MeV}$ to $\mathrm{GeV}$ energies by various space-born instruments $[1,2,3]$, lately in beautiful detail by the Fermi-LAT instrument [4]. At these energies, the diffuse Galactic emission dominates the $\gamma$-ray sky and outshines the individual $\gamma$-ray sources. Going to higher energies of $\mathrm{GeV}$ to $\mathrm{TeV}$ (and therefore probing higherenergy cosmic rays) the picture changes and the diffuse emission becomes subdominant. This is a consequence of the energy dependence of diffusion, resulting in a spectrum that is steeper for the diffuse $\gamma$-ray emission than for $\gamma$-ray sources. The very faint large-scale diffuse emission at $\mathrm{TeV}$ energies has for the first time been seen in the data of the H.E.S.S. Galactic plane survey (HGPS) $[5,6]$. This measurement was performed in regions of no significant $\gamma$-ray detection in the HGPS data and reveals an accumulated excess towards the Galactic equator.

Interpretation of this measurement has been challenging for various reasons. First of all, the study of cosmic-ray propagation by the means of diffuse $\gamma$-ray emission is generally a complex endeavour due to the various contributing components and their dependence on in parts poorly known Milky Way properties like the distribution of gas and the Galactic radiation field. Additionally, sophisticated codes for the propagation of cosmic rays are needed to induce properties of cosmic rays. While a lot of progress has been made here in recent years $[8,9,10]$, a further complication becomes apparent at $\mathrm{TeV}$ energies: the contribution of unresolved $\gamma$-ray sources plays a major role and needs to be disentangled from the "truly diffuse" emission stemming from the interaction of freely propagating cosmic rays. Unresolved sources comprise all $\gamma$-ray sources that lie under the current detection threshold of the observing instrument because they are either not luminous enough or too distant to be significantly detected. A diffuse signal is produced if a large number of unresolved sources contributes with individually weak emission. While it has already been argued that unresolved sources play a role also at energies of a few $\mathrm{GeV}[11,12]$, at $\mathrm{TeV}$ energies it is likely to dominate over the "truly diffuse" emission component.

In this work, the contribution of unresolved sources is investigated in the diffuse emission signal measured by H.E.S.S. For this purpose, a population of VHE $\gamma$-ray sources is simulated and constrained by the requirement to describe the known $\gamma$-ray sources of the HGPS in the region of completeness, i.e. at fluxes that can be significantly detected throughout the region of the HGPS.

\section{Simulation of a VHE source population}

\subsection{Construction of a model for VHE source population}

In order to infer properties of the $\gamma$-ray source population as a whole and especially the part hidden under the detection threshold of current instruments, assumptions need to be invoked to construct a model, which can then be used to "extrapolate" our knowledge of measured $\gamma$-ray sources to the domain of unresolved sources. The model that is used here is a simplistic one 


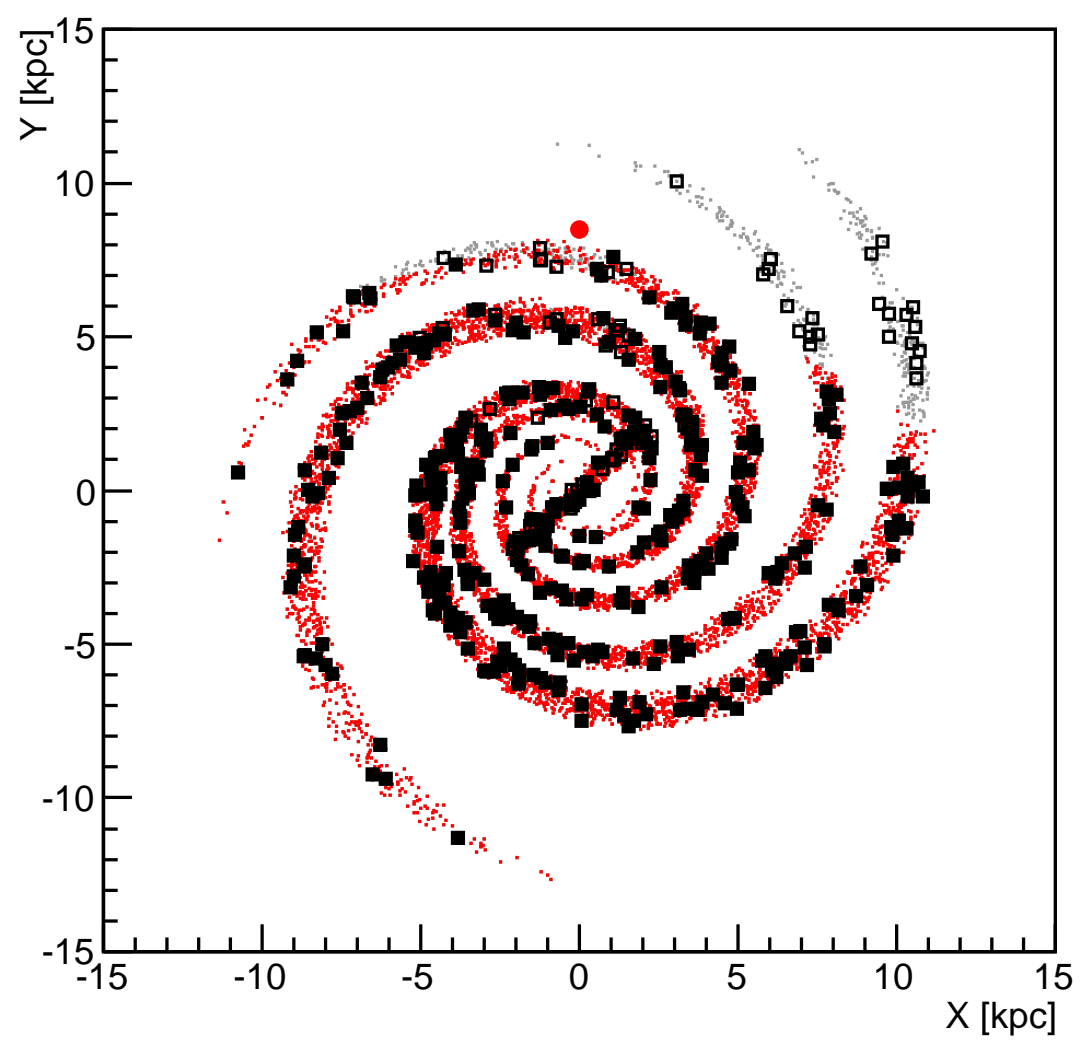

Figure 1: Head-on view of the simulated Milky Way with a four-arm structure and central bar. Dots are a high-statistics simulation with a total of 10,000 sources. Red markers denote the sources that fall into the investigated HGPS region, grey ones lie outside this region. Also shown is a realisation with limited statistics of 500 simulated sources (black squares), lying in the HGPS region (filled squares) or outside of the HGPS region (empty squares). The position of the Earth is marked with a red circle at the position $(0$, 8.5) $\mathrm{kpc}$.

mostly based on the geometry of the Milky Way. $\gamma$-ray sources are simulated with four basic parameters, three characterising their spatial position and one describing their luminosity at $1 \mathrm{TeV}$. The spatial distribution of the $\gamma$-ray sources is assumed to follow the spiral arms of the Milky Way, or more precisely, their matter content. The rationale behind this assumption is that stars form in dense regions and evolve to later form a supernova remnant or pulsar wind nebula (which is only an approximation: neither is the star formation rate of massive stars a one to one mapping of the content of interstellar matter nor is the region of star formation necessarily the place of $\gamma$-ray emission - $c f$. the offset of pulsar wind nebulae). The model adopted for the distribution of spiral arms is that of [13], more specifically, their four-arm emissivity model traced by CII. In addition, the simulation contains a central bar, as observed in Spitzer data [14].

In order to determine the spread in the simulations, a total of 1000 Milky Way representations is produced. Every representation contains a total of 500 sources. The distribution of sources can be seen in Fig. 1 for the case of a high-statistics simulation with 10,000 sources and one example of a 
Milky Way representation with 500 sources.

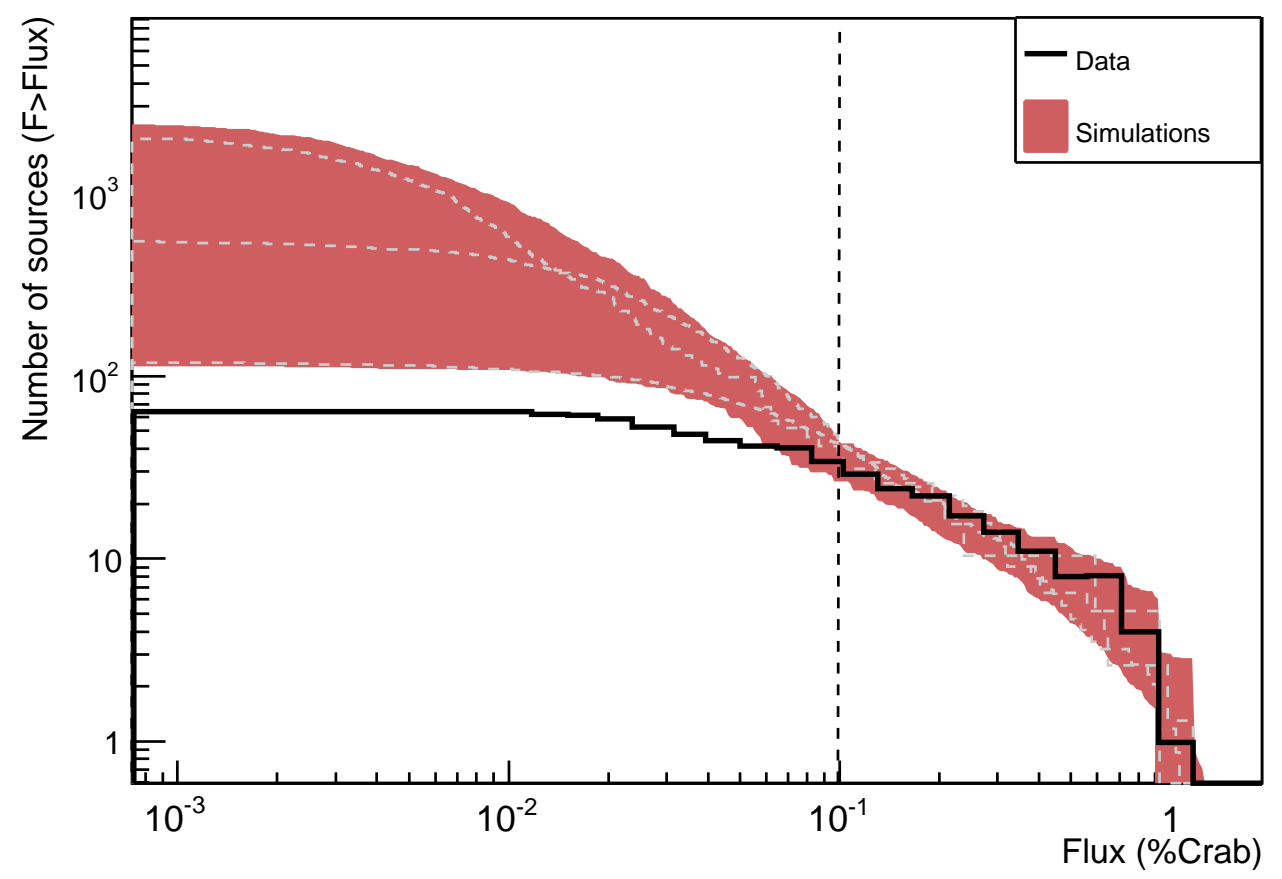

Figure 2: The $\log N-\log S$ distribution of the known HGPS sources, reproduced from [7] (black solid line) together with the $\log N-\log S$ band obtained from simulations (red area). The $\log N-\log S$ of three simulated Milky Way realisations are shown exemplarily as grey dashed lines. The completeness threshold at $10 \% \mathrm{Crab}$ is marked with a vertical dashed line.

\subsection{Constraining the simulations with measured $\gamma$-ray sources}

In order to derive the amount of unresolved sources in the H.E.S.S. diffuse emission, the simulation of the Galactic VHE $\gamma$-ray source population needs to be matched to reality, i.e. to be compared to the measured VHE $\gamma$-ray sources. In the simplest approach, only the flux of the sources is considered. In this case, the population is characterised by the distribution of the number count of sources with fluxes above a certain flux threshold as function of flux threshold, the $\log N-\log S$ distribution. In order to allow the comparison with the H.E.S.S. diffuse emission measurement, in every simulated Milky Way realisation only those sources are selected that fall into the range of the region that was used for the diffuse emission measurement [5], namely a longitude range of $-75^{\circ}<l<60^{\circ}$. Because the H.E.S.S. diffuse signal is observed in a latitude range of $-1^{\circ}<b<1^{\circ}$ also the simulations are constrained to this latitude range. For simplicity, the complex structure of excluded significant $\gamma$-ray emission (see Fig. 1 in [5]) is not reproduced; the effect is assumed to be subdominant. The sources that fall into this longitude and latitude range are highlighted in Fig. 1 (note that in the two-dimensional presentation few sources, especially nearby ones, are not included although they seem to fall into the right range; this is due to the small opening angle of $\pm 1^{\circ}$ in latitude). The $\log N-\log S$ distribution of the simulations is produced by calculating the flux measured at Earth for those sources that fall into the selected area, and counting. 
To constrain the simulations by the existing data of VHE $\gamma$-ray sources from the HGPS, the $\log N-$ $\log S$ distribution of the HGPS sources is needed. The only published $\log N-\log S$ distribution of the HGPS up to date can be found in [7] and is reproduced in Fig. 2. The distribution fully describes the underlying population of VHE $\gamma$-ray sources in the range of completeness, i.e. the range of fluxes above the detection threshold of the considered region. In [7] the completeness threshold is quoted to be $10 \%$ Crab, indicated by the dashed line in the figure. Due to the inhomogeneous exposure of the HGPS there is a significant number of sources below this threshold, stemming from regions with deeper exposure. It should be mentioned that the choice of this $\log N-\log S$ distribution introduces an inconsistency in the presented procedure: for the definition of the diffuse analysis region of [5] more recent data has been used, where some previously unresolved emission was signficantly detected and thus excluded from the diffuse emission measurement. This results in a bias towards larger contributions of unresolved sources in the presented framework.

In order to have the simulations describe the real world as seen in the HGPS, every simulated Milky Way realisation is fit to the $\log N-\log S$ of H.E.S.S. sources in the range of completeness. The fit is performed in both $x$ and $y$ coordinates, i.e. both the flux and the number of sources is adjusted to best describe the existing data. Therefore, the assumption on the level of the luminosity has no impact on the result, only the simulated spread in luminosities influences the shape of the $\log N-\log S$ distribution. Similarly, the fixed number of simulated sources of 500 enters only in the magnitude of the statistical fluctuations, the total number of sources is determined from the fit to the $\log N-\log S$ distribution. A large fraction of simulations fail to describe the measured $\log N-\log S$ distribution completely and are therefore discarded from the further analysis. In order to determine the number and the total flux of the unresolved sources together with the associated errors, the median and the scatter of the fitted simulations is determined for every flux bin. The $68 \%$ containment around the median value is shown by the red band in Fig. 2.

\section{Results and Discussion}

The difference between the HGPS data and the simulations below the completeness limit of $10 \%$ Crab indicates the amount of unresolved emission that is present in the data set. When it comes to sources with very low fluxes far off the completeness limit, the spread in the simulations increases as small deviations at the completeness threshold get magnified with the large lever arm. This spread results in a huge variation of the total number of sources between 115 and 2500, with the median still being at around 500. Significantly smaller is the spread in the total flux of unresolved sources. Taking into account the solid angle for which the $\log N-\log S$ distribution was obtained, the flux in unresolved emission accounts to $300 \%$ of the measured diffuse signal for the median. The lower limit of the simulations indicates that at least $2 / 3$ of the measured diffuse signal is due to unresolved sources. The overshooting of the estimation can at least partly be explained by the earlier mentioned inconsistency between the number of sources that enter the used $\log N-\log S$ distribution on the one hand, and the number of sources excluded for the diffuse emission measurement on the other hand.

Despite being simplistic the presented model provides a good guess of the behaviour of VHE $\gamma$-ray sources in the limit of low fluxes and what to expect of next generation instruments with increased sensitivity and reduced completeness threshold. Since VHE $\gamma$-ray sources are not that vastly abun- 
dant, any more precise model will always suffer from large uncertainties due to statistical fluctuations. Major improvements here can only be obtained by significant reduction of the detection threshold, which increases the lever arm and reduces the range of low fluxes that the simulations have to bridge with no data constraint.

Several possibilities exist to improve the current simulation scheme. A clear improvement in the presented method is to be expected by using a $\log N-\log S$ distribution that corresponds to the $\gamma$ ray sources that have been excluded for the diffuse emission measurement, which will be available with the upcoming H.E.S.S. catalog of Galactic sources. This will mitigate the current inconsistency in the definition of detected sources, and potentially also reduce the simulation overshoot over the data. Additionally, further studies are needed on the stability of the result under variation of the underlying assumptions.

The inclusion of spatial extension of the $\gamma$-ray sources is likely to have an effect on the detectability of nearby and very extended sources. In conjunction with extended sources also latitudinal and longitudinal source distributions can be used to apply additional constraints to the simulations. This is also important for constructing a template of unresolved-source emission, which might be relevant for advanced analysis methods beyond the standard background subtraction that was applied in [5], like a template-based log-likelihood fitting of the data. The discrimination between source classes with different spatial and luminosity distributions might also be a possibility to describe the existing data more accurately.

\section{References}

[1] G. F. Bignami, C. E. Fichtel, D. A. Kniffen, D. J. Thompson, High-energy galactic gamma radiation from cosmic rays concentrated in spiral arms, Astrophys. J. 199 (1975) 54.

[2] H. A. Mayer-Hasselwander et al., Large-scale distribution of galactic gamma radiation observed by COS-B, A\&A 105 (1982) 164-175.

[3] S. D. Hunter et al., EGRET Observations of the Diffuse Gamma-Ray Emission from the Galactic Plane, Astrophys. J. 481 (1997) 205.

[4] M. Ackermann et al. (Fermi-LAT Collaboration), Fermi-LAT Observations of the Diffuse $\gamma$-Ray Emission: Implications for Cosmic Rays and the Interstellar Medium, Astrophys. J. 750 (2012) 3.

[5] A. Abramowski et al. (H.E.S.S. Collaboration), Diffuse Galactic gamma-ray emission with H.E.S.S., PRD 90 (2014) 122007.

[6] S. Carrigan et al. for the H.E.S.S. Collaboration, The H.E.S.S. Galactic Plane Survey - maps, source catalog and source population in proceedings of 33rd International Cosmic Ray Conference, Rio de Janeiro (2013).

[7] M. Renaud, Latest results on Galactic sources as seen in VHE gamma-rays in Proceedings of 44th Recontres de Moriond (2009).

[8] https://galprop.stanford.edu/

[9] C. Evoli, D. Gaggero, A. Vittino, G. Di Bernardo, M. Di Mauro, A. Ligorini, P. Ullio, D. Grasso, Cosmic-ray propagation with DRAGON2: I. numerical solver and astrophysical ingredients, JCAP 02 (2017) 015. 
[10] R. Kissmann, PICARD: A novel code for the Galactic Cosmic Ray propagation problem, Astropart. Phys. 55 (2014) 37-50.

[11] A. W. Strong, Source population synthesis and the Galactic diffuse gamma-ray emission, Astrophysics and Space Science 309 (2007) 35.

[12] H. J. Völk and E. G. Berezhko, On The Fermi-Lat Surplus of the Diffuse Galactic Gamma-Ray Emission, Astrophys. J. 777 (2013) 149.

[13] T. Y. Steiman-Cameron, M. Wolfire, D. Hollenbach, COBE and the galactic interstellar medium: geometry of the spiral arms from FIR cooling lines, Astrophys. J. 722 (2010) 1460.

[14] E. Churchwell et al., The Spitzer/GLIMPSE Surveys: A New View of the Milky Way, Publications of the Astronomical Society of the Pacific 121 (2009) 213. 